\title{
Individual differences in stored digram frequencies and the immediate serial recall of letter and number strings'
}

\author{
M. S. MAYZNER AND M. E. TRESSELT \\ NEW YORK UNIVERSITY
}

This study examined the immediate serial recall of letter and number strings as a joint function of (a) the single-letter and sequential digram frequencies of the letter strings and (b) individual differences in the degree to which Ss already have in "store" information conceming the relative frequency of digrams as they occur in the language. The results clearly showed that high digram storage Ss show superior recall to low digram storage Ss for letter strings that vary both with respect to their single-letter frequencies and sequential digram frequencies, but that no differences in recall occur between the high and low digram storage Ss for the number strings.

In a previous study (Mayzner \& Schoenberg, 1964) the effects on immediate serial recall of single-letter frequencies and sequential digram frequencies were examined for letter strings composed of (1) high frequency letters and high frequency sequential digrams (HH), (2) high frequency letters and low frequency sequential digrams (HL), and (3) low frequency letters and low frequency sequential digrams (LL). The results, predicted on the basis of the "spew hypothesis" of Underwood \& Schulz (1960), were confirmed and showed recall to be best for the $\mathrm{HH}$ strings, intermediate for the HL strings, and poorest for the LL strings.

In the present study, individual differences in the degree to which Ss presumably already have in "store" information concerning the relative frequency of digrams as they occur in English is assessed by means of a skeleton-word digram ranking test (Mayzner \& Tresselt, 1962). This 60-item test with scoring key and norms may be found in a recent study by Tresselt \& Mayzner (1966). It is predicted, again on the basis of the "spew' hypothesis," that Ss who score high on this test, and therefore already have in storage a hierarchy of responses which accurately reflects digram frequencies as they occur naturally in the language, should yield better recall scores on letter strings than Ss who score low on this test. It is also predicted that no differences in recall performance on number strings should occur between high and low test scorers, if the test is truly reflecting letter transition probability habits and not merely general "memory ability."

Method

One hundred and thirty students in introductory psychology classes at New York University were administered the skeleton-word digram ranking test (Tresselt \& Mayzner, 1966) and $50 \mathrm{Ss}$, a high storage group (HS) with scores ranging from 19 to $27(\mathrm{~N}=25)$ and a low storage group (LS) with scores ranging from 9 to $18(\mathrm{~N}=25)$, were selected for the presentation of 18 letter strings and six number strings. Half the Ss received the letter strings first and the other half the number strings first. The number strings consisted of the nine digits (1 through 9) each used once, typed in six different random orders on six $3 \times 5$ in. cards, in a single horizontal line. The 18 letter strings (each nine letters in length, typed in a single horizontal line) were identical with those used in the previously cited study (Mayzner \& Schoenberg, 1964), and consisted of six $\mathrm{HH}$ strings, six HL strings, and six LL strings. Each $S$ received the 18 letter strings in a different random order, with the constraint that the first, second, and third block of six trials each contained two $\mathrm{HH}$, two $\mathrm{HL}$, and two LL strings. Each of the 24 cards (six number strings and 18 letter strings) were displayed to $S$ for $6 \mathrm{sec}$. and $S$ was then given $10 \mathrm{sec}$. to write the numbers or letters down. For each recall, $S$ was given a blank card with nine dashes on it and was told to write down the letters or numbers he could recall in their correct position and sequence.

\section{Results and Discussion}

For the letter strings the number of correct letters (i.e., the correct letter in the correct position) was summed across each group of six strings and the means were as follows, with the standard deviations in parentheses: Conditions HS-HH-45.44 (5.71); HSHL-37.08 (7.96); HS-LL-24.36 (4.86); LS-HH-36.00 (9.09); LS-HL-29.04 (8.08); and LS-LL-21.08 (5.11). Application of a 2 by 3 mixed analysis-of-variance design (Edwards, 1960, p. 227) to the above results yielded an $F=18.2, \mathrm{df}=1 / 48, \mathrm{p}<.001$ for the HS-LS main effect, an $F=170.7$, df $=2 / 96, p<.001$ for the $\mathrm{HH}-\mathrm{HL}-\mathrm{LL}$ main effect, and an $\mathrm{F}=5.5, \mathrm{df}=2 / 96, \mathrm{p}<.01$ for the interaction term, and application of t-tests to the three HS-LS comparisons for the HH, HL, and LL strings yielded t's of 4.30, 3.47, and 2.28 , and $\mathrm{p}$-values $<.01,<.01$, and $<.05$, respectively. These findings fully confirm our earlier results (Mayzner \& Schoenberg, 1964), showing recall to be best with the $\mathrm{HH}$ strings, intermediate with the HL strings, and poorest with the LL strings. They also clearly show 


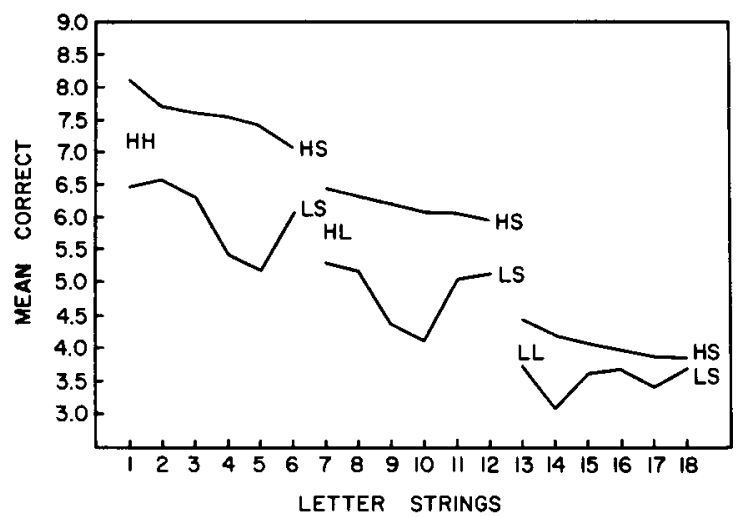

Fig. 1. Nean number of letters correct for the high and low storage conditions (HS-LS) for the HH, HL, and LL letter strings.

the highly significant effect of the HS-LS variable for all three types of letter strings, however, the significant interaction term suggests that the effect of the "storage" variable is strongest with the HH strings and weakest with the LL strings. These trends may be seen more clearly in Fig. 1, which shows the mean number of correct letters for each of the 18 letter strings for the HS and LS groups separately. It should be noted that the systematic downward trend of the means associated with the HS condition merely reflects the arbitrary plotting of the mean values for the 18 letter strings and nothing intrinsically systematic with respect to the experimental conditions.

In contrast to the results obtained with the letter strings, the number strings show no significant difference between the HS and LS groups; the mean number of correct digits (i.e., the correct digit in the correct position) for the HS and LS groups being 41.60 and 41.48 , respectively. These results strongly suggest that the digram ranking test is, in fact, assessing letter transition probability habits and not merely general "memory ability." Since a previous study (Tresselt \& Mayzner, 1965) had shown that scores on this digram ranking test also predict the speed with which Ss solve anagrams, the findings of the present study further indicate the usefulness of this test in assessing letter transition probability habits and their effects in a variety of relevant task situations. The overall results again are in accord with the "spew hypothesis" of Underwood \& Schulz (1960) and indicate that the digram ranking test might prove to be a useful technique in tapping underlying "storage" mechanisms implicated in the operation of the "spew hypothesis," as it relates to the storage and retrieval of letter strings.

\section{References}

Edwards, A. L. Elpcrimental design in psychological research. (Rev, ed.) New York: Rinehart, 1960.

Mayzner, M. S., \& Schoenberg, K. M. Single-letter and digram frequency effects in immediate serial recall. J. verbal Lcarn. verbal Behav., 1964, 3, 397-400.

Mayzner, M. S., \& Tresselt, M. E. The ranking of letter pairs and single letters to match digram and single-letter frequency counts. J. verbal Learn. verbal Bchav., 1962, 1, 203-207.

Tresselt, M. E., \& Mayzner, M. S. Anagram solution times: A function of individual differences in stored digram frequencies. $J$. cxp. Psychol., 1965, 70, 606-610.

Tresselt, M. E., \& Maymer, M. S. Normative solution times for a sample of 134 solution words and 378 associated anagrams. Psychon. Monogr. Suppl., 1966, 1, 293-298.

Underwood, B. J., \& Schulz, R. W. Mcaningfulness and verbal learning. Philadelphia: Lippincott, 1960.

\section{Note}

1. This research was supported in part by Contract Nonr 285(56) between the Engineering Psychology Branch of the Office of Naval Research and New York University. 